\title{
STATE OF ART AND CHALLENGES FOR THE CALCULATION OF RADIATIVE AND TRANSPORT PROPERTIES OF THERMAL PLASMAS IN HVCB
}

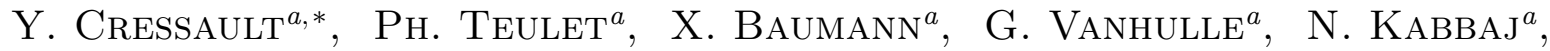 \\ F. Reichert ${ }^{b}$, A. PetchankA ${ }^{b}$ \\ ${ }^{a}$ (Laboratoire Plasma et Conversion d'Energie), Universite de Toulouse, CNRS, UPS, INPT, 118 route de \\ Narbonne, F-31062 Toulouse, France \\ b SIEMENS AG, Nonnendammallee 104, Berlin, 13629, Germany \\ * yann.cressault@laplace.univ-tlse.fr
}

\begin{abstract}
This paper is focused on the state-of-the-art and challenges concerning the thermophysical properties of thermal plasmas used in numerical modelling devoted to high voltage circuit breakers. For Local Thermodynamic Equilibrium (LTE) and Non-Local Thermodynamic (NLTE) and/or Chemical Equilibrium (NLCE) plasmas, the methods used to calculate the composition, thermodynamic, transport and radiative properties are presented. A review of these last data is proposed and some comparisons are given for illustrations.
\end{abstract}

Keywords: HVCB, chemical composition, transport coefficients, radiative properties, NLTE.

\section{Introduction}

Due to the cost of the experimental tests for HVCB, more and more numerical modelling is developed in order to compare the interruption capability of gases or mixtures with lower ODP than $\mathrm{SF}_{6}\left(\mathrm{CF}_{3} \mathrm{I}, \mathrm{CO}_{2}\right.$, $\mathrm{C}_{4} \mathrm{~F}_{7} \mathrm{~N}$ with Air, $\mathrm{N}_{2}$ or $\mathrm{O}_{2}$ ), to optimize the efficiency of the process by using new designs, new electrodes or new walls, or simply to better understand the physical and chemical processes in the plasma.

To develop the simulation of a high voltage circuit breaker using magneto-hydro-dynamic models (MHD), it is necessary to elaborate databanks of fundamental properties: the thermodynamic properties, the transport coefficients, and the radiative properties, these data being used in the different equations of conservation: mass conservation, momentum conservation and energy conservation. Depending on the region of the plasma (near the electrodes, near the walls, in the surrounding gases, in the core of the plasma), depending on the pressure and the temperature, depending on the intensity or power (ignition of the arc or its cooling and extinction), presence of turbulence or not, the Local Thermodynamic Equilibrium (LTE) hypothesis can be assumed or not when non-equilibrium effects appears. Consequently, the numerical simulations have to be performed considering nonequilibrium phenomena, which are done using the two-temperature assumption. In this we usually distinguish the electron kinetic temperature $T_{\mathrm{e}}$ which characterizes the high temperatures area of the plasma, while the heavy particles kinetic temperature $T_{\mathrm{h}}$ describes low temperature regions.

This paper tries to make a state of the art and to present the challenges on the calculation of the properties of plasmas used in HVCB, in the case of LTE and non-LTE assumptions. The first part is devoted to properties of plasmas in LTE conditions with a summarize of the gases studied as alternative gases or mixtures to $\mathrm{SF}_{6}$ and of the methods used to estimate the composition, the thermodynamic, transport and radiative properties. As the expressions available in the literature are accepted by the community in the case of LTE assumption, we will remember the recent works done on new mixtures and radiative properties. The second part is focused on the 2T-properties of plasmas in non-local thermodynamic equilibrium (nLTE) and/or non-local chemical equilibrium (nLCE). Few works have been done on this topic and lot of questions remain. We make a brief overview of the recent papers dealing with the $2 \mathrm{~T}$-properties applied to HVCB and finish the paper by indicating some challenges to solve.

\section{Plasma in LTE conditions}

For the HVCB applications, the first studies were devoted to the description of the heat zone, corresponding to the ignition of the arc in the first instants after the opening of the contacts. The plasma is assumed to be in Local Thermodynamic Equilibrium, the thermal properties are calculated with or without metallic vapors coming from the erosion of the electrodes $(\mathrm{Cu}, \mathrm{W}, \mathrm{CuW})$, with or without thermoplastic vapors coming from the erosion of the nozzle $\left(\mathrm{C}_{2} \mathrm{~F}_{4}\right)$. The LTE properties have been calculated for lots of pure gases or mixtures $\left(\mathrm{SF}_{6}, \mathrm{~N}_{2}\right.$, Air, $\mathrm{CO}_{2}, \mathrm{CF}_{3} \mathrm{I}$ $[1,2], \mathrm{CF}_{4}, \mathrm{C}_{3} \mathrm{~F}_{8}, \mathrm{C}_{4} \mathrm{~F}_{8}, \mathrm{c}-\mathrm{C}_{4} \mathrm{~F}_{8}, \mathrm{C}_{4} \mathrm{~F}_{7} \mathrm{~N}, \mathrm{C}_{4} \mathrm{~F}_{8} \mathrm{~N}_{2}$, $\mathrm{C}_{5} \mathrm{~F}_{10}, \mathrm{C}_{6} \mathrm{~F}_{12} \mathrm{O}, \mathrm{CF}_{3} \mathrm{NSF}_{2}, \mathrm{SF}_{6}-\mathrm{N}_{2}, \mathrm{SF}_{6}-\mathrm{CO}_{2}, \mathrm{SF}_{6}-$ $\mathrm{CF}_{4}, \mathrm{C}_{2} \mathrm{~F}_{4}-\mathrm{CO}_{2}, \mathrm{C}_{4} \mathrm{~F}_{8}-\mathrm{CO}_{2}$ [3-5], $\mathrm{CO}_{2}-\mathrm{N}_{2}-\mathrm{Cu}$ [6], $\mathrm{SF}_{6}-\mathrm{Cu}[7-9], \mathrm{C}_{5} \mathrm{~F}_{10} \mathrm{O}-\mathrm{CO}_{2}-\mathrm{O}_{2}$ [10]. The plasma composition is the first and unavoidable step allowing 
the computation of these properties, and is obtained from the law of mass action and the chemical base concept defined by Godin and Trépanier [11]. This technique is derived from the laws of thermodynamics and condensed phases are sometimes considered in the calculation of the population densities. Virial [12] and Debye [13] pressure corrections are often considered at high pressure $(P>10$ bars $)$ where the perfect gas law has to be replaced by a real gas law.

The thermodynamic properties (mass density, enthalpy, and specific heat at constant pressure) are directly deduced from the plasma compositions. Some results are given in [2-9] and behaviors are explained versus temperature, mixtures and pressure. The transport coefficients (viscosity, thermal and electrical conductivities) are obtained according to the ChapmanEnskog method and a previous calculation of collision integrals depending on the interaction potentials chosen to characterize the different collisions between particles (neutral-neutral, ion-neutral, electron-neutral and charged-charged collisions). The study of the collision integrals constitutes the most important part of the calculation of the transport coefficients and are often responsible for the differences observed between the authors [2-13].

The radiative losses play an important role in the HVCB arc plasmas: this is the main term of energy losses in the central region of the arc, the absorption in the surrounding plasma and cold gas leads to an increase of the pressure, it is responsible of the PTFE ablation influencing the behavior and the extinction of the plasma. The divergence of the flux is obtained by solving the radiative transfer equation which depends on the wavelengths, directions, and the geometry of the plasma. Due to the complexity of this equation, several simplifying approaches have been developed during the last decades such as the method of the net emission coefficient (NEC) initially proposed by Lowke and first calculated for $\mathrm{SF}_{6}$ by Liebermann and Lowke [14, 15], then by Aubrecht and Barlova or Gleizes et al. [16, 17]. In order to treat efficiently the radiative transfer in the middle or cold regions, the P1-Model or discrete ordinates methods are often preferred using grey body approximation or Mean Absorption Coefficients (MACs) [18-22]. Then, due to rise of the computer's capacity, some works have been developed on pure $\mathrm{SF}_{6}$ in order to perform an exact calculation of the radiative transfer equation with a very fine description of the spectrum, evaluating the accuracy and the validity of the simplified methods by comparisons. A quick review has been presented during the FSO conference in 2011 by A. Gleizes et al. [23]. Since, more studies on the radiative transfer equation have been done for pure gases $\left(\mathrm{SF}_{6}, \mathrm{~N}_{2}\right.$, Air, $\left.\mathrm{CO}_{2}\right)$, binary mixtures $\left(\mathrm{SF}_{6}-\mathrm{C}_{2} \mathrm{~F}_{4}, \mathrm{C}_{4} \mathrm{~F}_{8}-\mathrm{CO}_{2}, \mathrm{CO}_{2}\right.$ $\mathrm{Cu}[3,24])$ and ternary mixtures $\left(\mathrm{SF}_{6}-\mathrm{C}_{2} \mathrm{~F}_{4}-\mathrm{Cu} / \mathrm{W}\right)$ in 1D-2D-3D geometry with imposed temperatures profiles [25-28]. An example is given in Figure 1 for the divergence of the flux for a $\mathrm{SF}_{6}$ pure plasma at

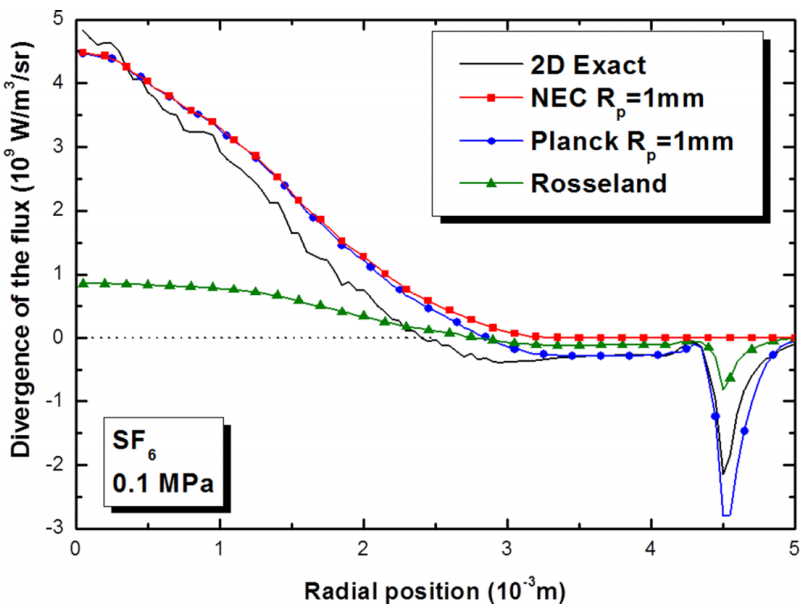

Figure 1. Divergence of the flux for a $S F_{6}$ pure plasma at $0.1 \mathrm{MPa}$, obtained with different approached methods [1].

1bar, obtained with different approached methods [1].

From these studies, some conclusions can be done: (1) for the equilibrium composition and thermodynamic functions, the methods are well-known and valid, maybe questionable for plasmas at very high pressure and low temperature; (2) for transport coefficients, the interaction potentials have a significant influence on the values obtained and validation is done through other parameters such as the pressure for the thermal conductivity. A very small concentration of metallic vapors $(>1 \%)$ strongly influences the electrical conductivity whereas viscosity and thermal conductivity are modified with concentration of metals or PTFE higher than 20-25\%. Consequently, it is important to consider those coefficients in the conservation equations if we want to study the plasma regions where the ablation/erosion of walls and electrodes is important; (3) for the radiative transfer, some differences can be observed on the NEC when estimated using the escape factor approximation instead of the line by line method (more than $40 \%$ of difference can be observed at very high pressure $>100$ bar [25]).

In LTE conditions, the challenges on thermal properties are mainly "driven" by the use of new alternative gases to $\mathrm{SF}_{6}\left(\mathrm{CO}_{2}, \mathrm{CF}_{3} \mathrm{I}, \mathrm{c}-\mathrm{C}_{4} \mathrm{~F}_{8}, \mathrm{C}_{4} \mathrm{~F}_{7} \mathrm{~N}, \mathrm{C}_{5} \mathrm{~F}_{10} \mathrm{O}\right.$, $\mathrm{C}_{6} \mathrm{~F}_{12} \mathrm{O}, \mathrm{CF}_{3} \mathrm{NSF}_{2}$, pure gases or mixed with $\mathrm{CO}_{2}$, $\mathrm{CF}_{4}, \mathrm{C}_{2} \mathrm{~F}_{4}, \mathrm{O}_{2}, \mathrm{~N}_{2}$, air) or by the use of new materials for the manufacturing of electrodes or nozzles $(\mathrm{Cu}, \mathrm{W}, \mathrm{CuW}$ which have an important impact on the electrical conductivity and the radiation even if in few concentration). Some numerical modelling have been developed to study the influence of the erosion of electrodes or ablation of nozzle on the arc behavior from its ignition to its extinction [29-34]. The radiative transfer calculation with simplified methods can be improved following four ways: a better treatment of the absorption coefficients for molecules [26]; a careful definition of the spectral intervals for the calculation 
of the Mean Absorption Coefficients as in their works of Jan et al., Norborg and Iordanidis or Kloc et al. $[26,27,35]$; the elaboration of MACs tables from a mix of the different mean functions (Planck averaging at high temperature considering the absorption of the lines, and mean natural value at low temperature with only continuum contribution) [26]; the use of an optimized function based on the minimization of the difference between the exact calculation of the radiative flux or the divergence of the flux and the results obtained with simplified methods [36-38].

\section{Plasma in nLTE and/or nLCE conditions}

In many plasma processes, departures from thermal or chemical equilibrium exist on the edges of the plasma and close to the walls and the electrodes. For example, in the case of breaking devices and during the current zero phase, the electron number density is not very significant, the energy transfer between the electrons and the heavy particles is not enough efficient to preserve thermal equilibrium. As a consequence, the electron kinetic temperature $T_{\mathrm{e}}$ is higher than the heavy particles temperature $T_{\mathrm{h}}$, and two-temperature models must be developed. The recent papers propose two equations of energy conservation: one for the electrons and the other for the heavy species [39]. These models require the computation of thermodynamic, transport and radiative properties as a function of $T_{\mathrm{e}}$ and $\theta=T_{\mathrm{e}} / T_{\mathrm{h}}$ and the first step to obtain these data is, as in the case of LTE, the calculation of the $2 \mathrm{~T}$ plasma composition.

There are several available theories in the literature to calculate the densities of species for non-equilibrium plasmas: (i) the minimization of a thermodynamic function (Gibbs'free enthalpy for plasma at constant pressure or Helmholtz's free energy at constant volume), (ii) the method based upon the $2 \mathrm{~T}$ mass action law (LMA) assuming the chemical equilibrium and coupled with a set of equations for the conservation of pressure, electrical charge and mixture proportions and (iii) collisional radiative models (CR) or a chemical kinetic model $(\mathrm{CK})$ coupled with an equation for the conservation of pressure. These methods give the same results in LTE but present strong differences if the plasma is not in equilibrium, depending on the temperatures affected the processes: the translatologie kinetic temperatures of electrons and heavy particles $T_{\mathrm{e}}$ and $T_{\mathrm{h}}$, the temperatures characteristic of the population of the various internal energy modes i.e. excitation temperature $T_{\text {ex }}$ associated to the population of excited electronic states (atomic and molecular), the vibration temperature $T_{\mathrm{v}}$ linked to the distribution of vibrational levels and the rotation temperature $T_{\mathrm{r}}$ related to the population of rotational states. The LMA method is relatively easy to implement in numerical codes, and requires few data (partition functions, enthalpies of formation at $0 \mathrm{~K}$ of each chemical species and mass of species). The collisional-radiative (CR)

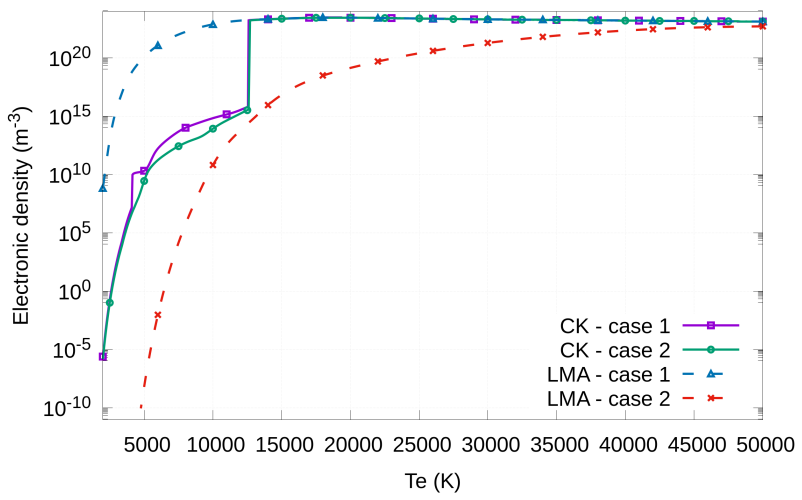

Figure 2. Electron number densities versus electronic temperature $T_{e}$ (CK: chemical kinetic model, LMA : law of mass action). Pure $S F_{6}, \theta=5, P=1$ bar.

and chemical kinetic (CK) models have advantages to reduce simplifying assumptions in the calculation. Moreover, the CK model allows studying the temporal chemical dynamic of the system. Nevertheless, a large databank of reaction rates or cross sections for each chemical process are needed. Relations between cross sections and reaction rates are well known for plasmas in thermal equilibrium conditions but are more complex for non-LTE plasmas. Indeed, electrons and heavy particles involved in chemical reactions have not the same temperature $\left(T_{\mathrm{e}}\right.$ and $\left.T_{\mathrm{h}}\right)$ and are not described statistically by the same distribution functions. The temporal evolution of densities for each chemical species is given by a set of master equations:

$$
\frac{\partial n_{i}}{\partial t}=C_{i}-n_{i} D_{i}
$$

where $C_{i}$ and $D_{i}$ are respectively the creation and destruction factors for the specie $i$. They depend on the densities of species participating at the creation/destruction of specie $i$ through the chemical reaction and on the reaction rates of chemical reactions involved. Using relations between forward and reverse reaction rates, we used the library DVODE to solve the system formed (Eq. 1). The recent works of the team compared these methods in the case of pure $\mathrm{SF}_{6}[40-42]$ and mixture $\mathrm{SF}_{6}-\mathrm{C}_{2} \mathrm{~F}_{4}$. We used two different assumptions concerning the population of internal energy modes: case (1) with $T_{\mathrm{ex}}=T_{\mathrm{e}}$, $T_{\mathrm{v}}=T_{\mathrm{e}}$ and $T_{\mathrm{r}}=T_{\mathrm{h}}$ more adapted to ionized gases (high temperatures), and case (2) $T_{\mathrm{ex}}=T_{\mathrm{h}}, T_{\mathrm{v}}=T_{\mathrm{h}}$ and $T_{\mathrm{r}}=T_{\mathrm{h}}$ more adapted to molecular gases (low temperatures). The figure 2 compares the electron number densities versus the electronic temperature $T_{\mathrm{e}}$ $\left(\theta=T_{\mathrm{e}} / T_{\mathrm{h}}=5\right)$, for pure $\mathrm{SF}_{6}$ at $1 \mathrm{bar}$, and obtained from the CK and LMA methods.

The thermodynamic properties of $2 \mathrm{~T}$ plasma are deduced from relations given Colombo et al. [43, 44] which propose to calculate a unique $2 \mathrm{~T}$ specific heat $C_{p}$ as the derivative of the total enthalpy $H_{\mathrm{tot}}=$ $H_{\mathrm{e}}+H_{\mathrm{h}}$ compared to the electronic temperature $T_{\mathrm{e}}$ : 


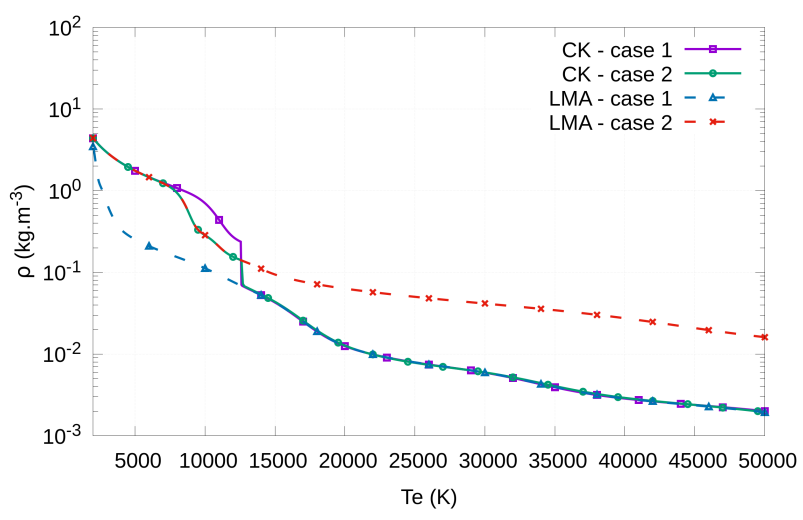

Figure 3. Mass density $\rho$ versus electronic temperature $T_{e}$ for pure $S F_{6}$ plasma, for $\theta=5$ and pressure of 1 bar.

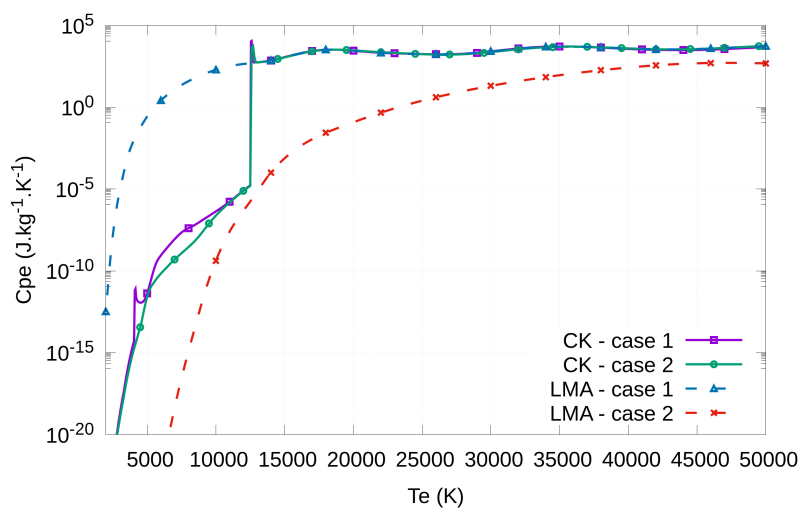

Figure 4. Specific heat at constant pressure of electrons $C_{P e}$ versus electronic temperature $T_{e}$ for pure $S F_{6}$, for $\theta=5$ and pressure of $1 \mathrm{bar}$.

$$
C_{P}\left(\theta, T_{e}\right)=\left(\frac{\partial H_{\mathrm{tot}}}{\partial T_{e}}\right)_{P}
$$

As energetic contributions of electrons and heavies are separated in numerical codes through two coupled energy conservation equations, it is necessary to calculate independently the enthalpy of electron $H_{\mathrm{e}}$ and heavy particles $H_{\mathrm{h}}$, the specific heats of electrons $C_{P \mathrm{e}}$ and heavy species $C_{P \mathrm{~h}}$ :

$$
\begin{aligned}
& C_{P \mathrm{e}}\left(\theta, T_{\mathrm{e}}\right)=\left(\frac{\partial H_{\mathrm{e}}}{\partial T_{\mathrm{e}}}\right)_{P} \\
& C_{P \mathrm{~h}}\left(\theta, T_{\mathrm{e}}\right)=\left(\frac{\partial H_{\mathrm{h}}}{\partial T_{\mathrm{h}}}\right)_{P}
\end{aligned}
$$

This separation of the specific heat in two distinct contributions for electrons and heavy species is also the approach retained by Ghorui et al. [45, 46] or Zhang et al. [47]. The figure 3 shows the mass density of pure $\mathrm{SF}_{6}$ obtained from the LMA and CK for the two cases, $\theta=5$ and 1 bar. The figures 4 and 5 present the two specific heats in the same conditions. The law of mass action is in dashed lines and the chemical kinetic method in full lines.

With the CK model, there is a transition between both cases of the LMA. We can conclude that the

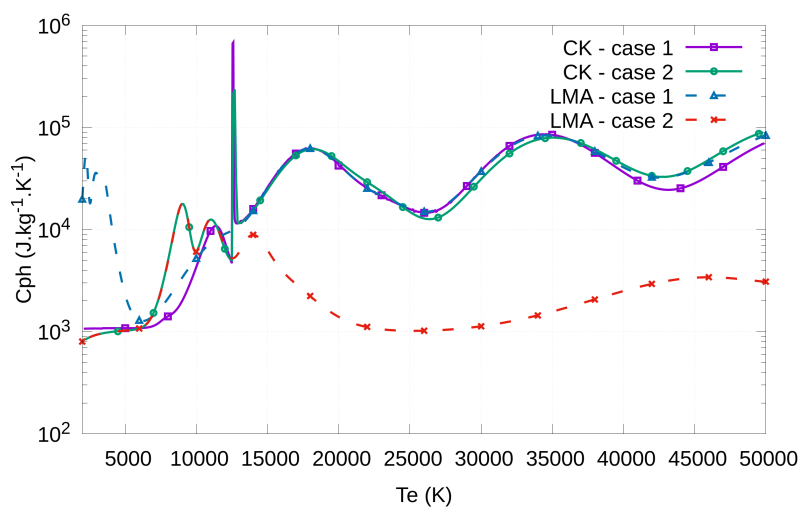

Figure 5. Specific heat at constant pressure of heavy particles $C_{P h}$ versus electronic temperature $T_{e}$ for pure $S F_{6}$, for $\theta=5$ and pressure of $1 \mathrm{bar}$.

LMA taken in the case 1 is accurate for the high temperatures and in the case 2 for the low temperature range. In high temperatures, there is lot of electrons and the inelastic processes are dominated by electron collisions and have to be calculated at $T_{\mathrm{e}}$ (case 1 ). In low temperatures, the heavy particles chemical processes are dominant and have to be calculated at $T_{\mathrm{h}}$ (case 2 ). There is a range of temperature where the LMA is not exact and the CK is necessary to calculate the composition.

Whereas the community agrees the calculation of the thermodynamic properties, translation thermal conductivities of electrons and heavy particles, viscosity and electrical conductivity, there is no consensus for the calculation of the reactive thermal conductivity. The theory of Hirschfelder et al. [12] or Bonnefoi et al. [48, 49] consider separately the electrons and the heavy particles whereas the theory of Rat showed that these two theories did not consider correctly not only the diffusion in $2 \mathrm{~T}$ plasmas but also the coupling between electrons and heavy particles. The Rat's theory leads to new definitions of collision integrals, transport coefficients and consequently new terms in energy fluxes. Ghorui et al. [45, 46] in 2008 and Colombo et al. in 2009 [43, 44] showed in their works that the method of Devoto is sufficient to determine the transport properties (electrical conductivity, thermal conductivity and viscosity) in $2 \mathrm{~T}$ assumption only if diffusion coefficients defined by Ramshaw were used. From the last works published on 2T-properties for several gases applied in various applications (Bonnefoi et al. [48, 49] for Ar-H, Mexmain [50] for Ar-O, McCourt [51] for polyatomic mixtures, Aubreton et al. [52-54] for Ar-O2, Ar-He or pure N2, Capitelli et al. $[55,56]$ with a "State-to-State" model applied for pure $\mathrm{N}_{2}$ and $\mathrm{H}_{2}$ plasmas, Guo et al. [57], Zhang et al. [58] and Yang et al. [59] on helium, argon and $\mathrm{SF}_{6}-\mathrm{N}_{2}$ mixtures) we can consider as accepted that the translation and internal thermal conductivities of heavy particles and the viscosity can be calculated at $T_{\mathrm{h}}$, the translation thermal conductivity of electrons and the electrical conductivity at $T_{\mathrm{e}}$. The figure 6 compare 
these coefficients obtained for the cases 1 and 2 , the LMA and CR/CK methods, in the case of pure $\mathrm{SF}_{6}$ at 1 bar and $\theta=5$. The computation of the $2 \mathrm{~T}$ reactive thermal conductivity at high pressure for complex gases such as $\mathrm{SF}_{6}$ using multi-temperature expressions from the literature (such as the one provided by Ghorui et al. [45, 46]) gave poor results. The use of the Ghorui's expressions seems to be accurate for pressure lower than 16 bar and pure gases $\left(\mathrm{O}_{2}, \mathrm{~N}_{2}\right)$ or mixtures with few chemical reactions $\left(\mathrm{O}_{2}-\mathrm{N}_{2}\right)$. For pure $\mathrm{SF}_{6}$ and $\mathrm{SF}_{6}-\mathrm{C}_{2} \mathrm{~F}_{4}$ mixtures, we extended the Butler and Brokaw theory to $2 \mathrm{~T}$ assumption. We divided the total reactive thermal conductivity into two contributions, one for the heavy particles (considering neutral atoms and molecules) and one for the electron part (considering electrons and ions):

$$
\kappa_{\text {reac }}^{\mathrm{h}}=-\frac{1}{R T_{\mathrm{h}}^{2}} \frac{\left|\begin{array}{cccc}
0 & \Delta h_{1} & \cdots & \Delta h_{N_{\mathrm{e}}} \\
\Delta h_{1} & A_{11} & \cdots & A_{N_{\mathrm{e}} 1} \\
\vdots & \vdots & \ddots & \vdots \\
\Delta h_{N_{\mathrm{e}}} & A_{1 N_{\mathrm{e}}} & \cdots & A_{N_{\mathrm{e}} N_{\mathrm{e}}}
\end{array}\right|}{\left|\begin{array}{cccc}
A_{11} & \cdots & A_{N_{\mathrm{e}} 1} \\
\vdots & \ddots & & \vdots \\
A_{1 N_{\mathrm{e}}} & \cdots & A_{N_{\mathrm{e}} N_{\mathrm{e}}}
\end{array}\right|}
$$

with

$$
\begin{aligned}
A_{i j} & =\frac{R T_{i j}}{P} \sum_{k=2}^{N_{\mathrm{e}}-1} \sum_{l=k+1}^{N_{\mathrm{e}}} \frac{x_{k} x_{l}}{D_{k l}^{b}}\left(\frac{\alpha(i, k)}{x_{k}}-\frac{\alpha(i, l)}{x_{l}}\right) \\
& \times\left(\frac{\alpha(j, k)}{x_{k}}-\frac{\alpha(j, l)}{x_{l}}\right)
\end{aligned}
$$

$T_{i j}$ is equivalent to $T_{\mathrm{e}}$ if one of the two particles in collision is an electron, $T_{\mathrm{h}}$ in the other cases. From the original expression of Butler and Brokaw, the temperature $T$ was replaced by $T_{\mathrm{e}}, T_{\mathrm{h}}$ or $T_{i j}$ in relations (4). This choice has been validated by comparisons of shape of the reaction thermal conductivities versus temperature, $\theta$ and pressure. As examples, the results of the electrical conductivity, viscosity, translational, reactive and total thermal conductivities of electron and heavy particles are presented in figure 6 for $50 \% \mathrm{SF}_{6}-50 \% \mathrm{C}_{2} \mathrm{~F}_{4}$ at 1 bar and $\theta=5$, obtained from CK and LMA methods. For the case 1, the reaction term for heavy particles increases with $\theta$, the contribution of electrons decreases. For the case 2, the values of $\kappa_{\text {reac }}^{\mathrm{h}}$ are shifted toward high temperatures and the peaks of $\kappa_{\text {reac }}^{\mathrm{e}}$ are diminished and shifted to higher temperatures [60].
Finally, the last part concerns the calculation of the $2 \mathrm{~T}$ radiative properties. For radiation in nLTE, some works have been done on atmospheric reentry by Soucasse et al. [61], or Scoggings [62] but few are available in the literature for plasmas used in HVCB. Contrarily to studies in LTE, the specialita emission and absorption coefficients cannot be linked in the Kirshhoff law through the Planck function. Recent papers propose to write two equations of conservation of energy: one for the electron with divergence of the flux obtained with the net emission coefficient approximation considering only the continuum part, another one for the heavy species with divergence of the flux obtained with the same approximation considering only the contribution of the lines. Consequently, the radiation has to be separated into two parts: one coming from atomic (radiative recombination, radiative attachment, Bremsstrahlung) and molecular continua and calculated at the temperature $T_{\mathrm{e}}$, and the other one coming from the atomic lines and calculated at $T_{\text {ex }}$ (consequently $T_{\mathrm{e}}$ or $T_{\mathrm{h}}$ depending on the case 1 or 2), the molecular lines being neglected at this step. The broadenings have been calculated assuming $T_{\mathrm{h}}$ for the FWHM due to the Doppler effect, $T_{\mathrm{h}}$ for the van der Waals effect, and $T_{\mathrm{e}}$ for the Stark perturbation. The correction proposed by Griem has been taken into consideration by calculating the parameter $R$ with $T_{\mathrm{h}}$. Then, two net emission coefficients can be estimated:

$$
\begin{aligned}
& \varepsilon_{N}^{\text {cont }}=\int_{0}^{+\infty} \varepsilon_{\lambda}^{\text {cont }}\left(\lambda, T_{\mathrm{e}}\right) e^{-R_{\mathrm{p}} \kappa_{\mathrm{tot}}^{\prime}\left(\lambda, T_{\mathrm{e}}\right)} \mathrm{d} \lambda \\
& \varepsilon_{N}^{\text {lines }}=\int_{0}^{+\infty} \varepsilon_{\lambda}^{\text {lines }}\left(\lambda, T_{\mathrm{ex}}\right) e^{-R_{\mathrm{p}} \kappa_{\mathrm{tot}}^{\prime}\left(\lambda, T_{\mathrm{ex}}\right)} \mathrm{d} \lambda
\end{aligned}
$$

Figure 7 shows the influence of $\theta$ on the NECs for the two cases. It means in our case that NEC for continuum is always dependent on $T_{\mathrm{e}}$ but NEC for atomic lines depend on $T_{\mathrm{e}}$ (case 1 ) or $T_{\mathrm{h}}$ (case 2 ). As the NEC for the continuum only depends on $T_{\mathrm{e}}$, the factor $\theta$ has no influence. This is not the same conclusion for the NEC of the atomic lines since $T_{\mathrm{ex}}$ is equal to $T_{\mathrm{e}}$ for the case $1\left(T_{\mathrm{ex}}=T_{\mathrm{e}}, T_{\mathrm{v}}=T_{\mathrm{e}}\right.$ and $\left.T_{\mathrm{r}}=T_{\mathrm{h}}\right)$ or $T_{\mathrm{h}}$ for the case $2\left(T_{\mathrm{ex}}=T_{\mathrm{h}}, T_{\mathrm{v}}=T_{\mathrm{h}}\right.$ and $\left.T_{\mathrm{r}}=T_{\mathrm{h}}\right)$.

\section{Conclusion}

We have presented the methods commonly used to determine the thermophysical properties (composition, thermodynamic, transport and radiative properties) for LTE and NLTE/NLCE plasmas existing in HVCB. For LTE plasmas, the methods are well-known, as the influence of the metallic vapors on the properties: a small proportion of metallic vapors (inferior to 1\%) strongly impacts the electrical conductivity and the radiation, higher proportions is required (more than $20 \%$ ) to modify significantly the thermal conductivity and the viscosity. The challenges will be not only the calculation of these properties for new materials 

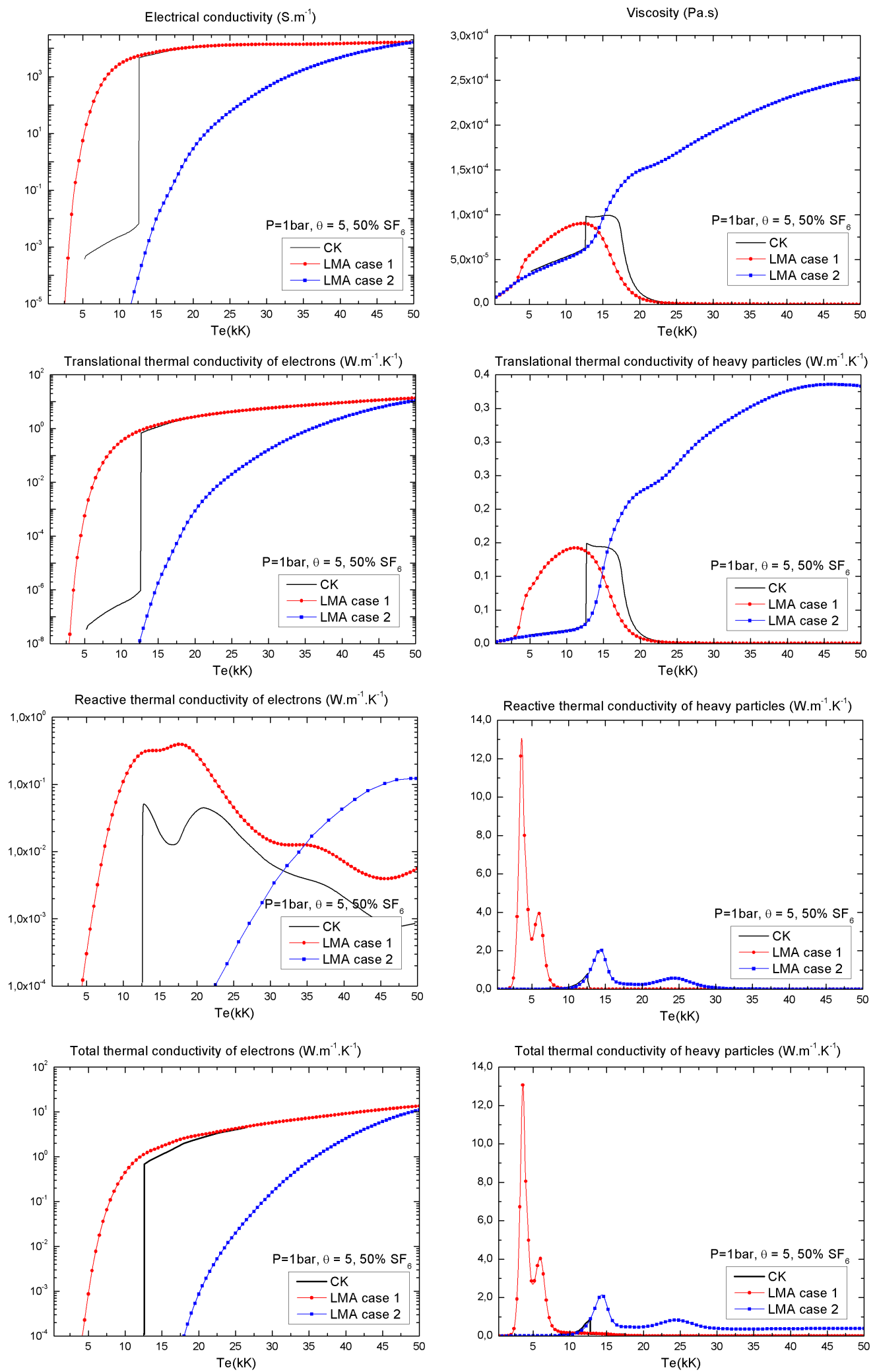

Figure 6. $2 T$ transport coefficients of $50 \% \mathrm{SF}_{6}-50 \% \mathrm{C}_{2} \mathrm{~F}_{4}$ (mass proportions) obtained with LMA, and CK methods, for $\theta=5$ and $1 \mathrm{bar}$. 


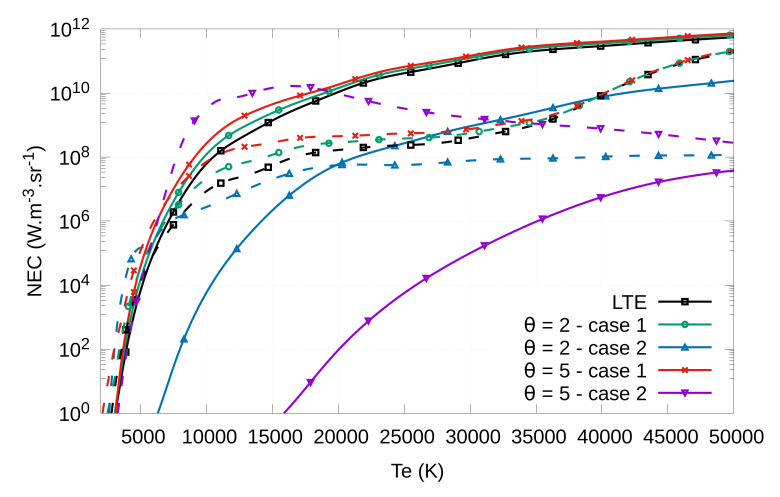

Figure 7. Influence of $\theta$ on the two NECs versus electron temperature for a pure $S F_{6}$ plasma at $1 \mathrm{bar}$ and for $R_{p}=1 \mathrm{~mm}$ : full lines for the atomic lines, dashed lines for the continuum.

(used for electrodes or nozzles) but also the treatment of the RTE solving based on fine discretization and better approached methods. For NLTE/NLCE plasmas in HVCB, lots of challenges persist and must be solved rapidly in order to contribute to the improvement of the MHD modelling. For the plasmas compositions, it will be necessary to develop more precise models such as collisional radiative models or chemical kinetic models which are (for the moment) complex to achieve due to the lack of the reaction rates or collisional cross-sections. For the transport properties, the community must consider the thermal reactional conductivity and answer to the questions: should we consider only one coefficient (only for heavy particles for example), two contributions, a total thermal conductivity and how to calculate them under these conditions of non-thermal/chemical equilibrium? For the radiative properties, some works have been developed on $\mathrm{CO}_{2}$ or air for re-entry atmosphere phenomena, syngas or combustion researches but few data are available for HVCB with or without metallic vapors due to the numerous chemical reactions. As the Planck and Kirchhoff laws are not accurate in non-equilibrium conditions, it is essential to treat differently the emission and absorption phenomena. The greatest difficulty lies in estimating correctly the populations energy levels, the emission and the absorption being proportional to these populations. The treatment of the atomic lines will be one of the main locks to raised (population of levels and broadenings being governed by different temperatures). New models will need to be developed to consider radiative phenomena separately: Hybrid Statistical Narrow Band model (HSNB) combining Statistical Narrow-Band model (SNB) for the optically thick molecular systems and Narrow Band means for the emission and absorption coefficients of the optically thin molecular systems and continua, a line by line (LBL) calculation for the bound-bound atomic lines [62]. Finally, these new methods and databank have to be validated with the comparison of MHD modeling results and experimental works.

\section{References}

[1] Y. Cressault. Basic knowledge on radiative and transport properties to begin in thermal plasmas modelling. AIP Advances, 5(5):057112, 2015. doi:10.1063/1.4920939.

[2] Y. Cressault, V. Connord, H. Hingana, P. Teulet, and A. Gleizes. Transport properties of $\mathrm{CF}_{3} \mathrm{I}$ thermal plasmas mixed with $\mathrm{CO}_{2}$, air or $\mathrm{N}_{2}$ as an alternative to $\mathrm{SF}_{6}$ plasmas in high-voltage circuit breakers. Journal of Physics D: Applied Physics, 44(49):495202, 2011. doi: $10.1088 / 0022-3727 / 44 / 49 / 495202$.

[3] L. Zhong, Y. Cressault, and P. Teulet. Thermophysical and radiation properties of high-temperature $\mathrm{C}_{4} \mathrm{~F}_{8}-\mathrm{CO}_{2}$ mixtures to replace $\mathrm{SF}_{6}$ in high-voltage circuit breakers. Physics of Plasmas, 25(3):033502, 2018. doi:10.1063/1.5012850.

[4] P. André and Z. Koalaga. Composition of a thermal plasma formed from ptfe with copper in non-oxidant atmosphere, Part I: Definition of a test case with the $\mathrm{SF}_{6}$. High Temperature Material Processes: An International Quarterly of High-Technology Plasma Processes, 14(3), 2010. doi:10.1615/HighTempMatProc.v14.i3.70.

[5] A. Yang, Y. Liu, B. Sun, X. Wang, Y. Cressault, L. Zhong, M. Rong, Y. Wu, and C. Niu. Thermodynamic properties and transport coefficients of hightemperature $\mathrm{CO}_{2}$ thermal plasmas mixed with $\mathrm{C}_{2} \mathrm{~F}_{4}$. Journal of Physics D: Applied Physics, 48(49):495202, 2015. doi:10.1088/0022-3727/48/49/495202.

[6] L. Zhong, X. Wang, M. Rong, and Y. Cressault. Effects of copper vapour on thermophysical properties of $\mathrm{CO}_{2}-\mathrm{N}_{2}$ plasma. The European Physical Journal D, 70(11):233, 2016. doi:10.1140/epjd/e2016-70241-3.

[7] M. Rong, L. Zhong, Y. Cressault, A. Gleizes, X. Wang, F. Chen, and H. Zheng. Thermophysical properties of $\mathrm{SF}_{6}-\mathrm{Cu}$ mixtures at temperatures of $300-30000 \mathrm{~K}$ and pressures of $0.01-1.0 \mathrm{MPa}$ : part 1. Equilibrium compositions and thermodynamic properties considering condensed phases. Journal of Physics D: Applied Physics, 47(49):495202, 2014. doi:10.1088/0022-3727/47/49/495202.

[8] X. Wang, L. Zhong, Y. Cressault, A. Gleizes, and M. Rong. Thermophysical properties of $\mathrm{SF}_{6}-\mathrm{Cu}$ mixtures at temperatures of $300-30,000 \mathrm{k}$ and pressures of 0.01-1.0 MPa: part 2. Collision integrals and transport coefficients. Journal of Physics D: Applied Physics, 47(49):495201, 2014. doi: $10.1088 / 0022-3727 / 47 / 49 / 495201$.

[9] B. Chervy, A. Gleizes, and M. Razafinimanana. Thermodynamic properties and transport coefficients in $\mathrm{SF}_{6}-\mathrm{Cu}$ mixtures at temperatures of $300-30000 \mathrm{~K}$ and pressures of 0.1-1 MPa. Journal of Physics D: Applied Physics, 27(6):1193, 1994. doi:10.1088/0022-3727/27/6/017.

[10] L. Zhong, M. Rong, X. Wang, J. Wu, G. Han, G. Han, Y. Lu, A. Yang, and Y. Wu. Compositions, thermodynamic properties, and transport coefficients of high-temperature $\mathrm{C}_{5} \mathrm{~F}_{10} \mathrm{O}$ mixed with $\mathrm{CO}_{2}$ and $\mathrm{O}_{2}$ as substitutes for $\mathrm{SF}_{6}$ to reduce global warming potential. AIP Advances, 7(7):075003, 2017. doi:10.1063/1.4993305. 
[11] D. Godin and J. Trépanier. A robust and efficient method for the computation of equilibrium composition in gaseous mixtures. Plasma chemistry and plasma processing, 24(3), 2004. doi:10.1007/s11090-004-2279-8.

[12] J. Hirschfelder, C. Curtis, and R. Bird. Molecular Theory of Gases and Liquids. Second printing. New York: Wiley, 1964.

[13] M. Boulos. Thermal plasmas: fundamental and applications vol 1. First printing. Springer-Verlag New York, 1994.

[14] J. Lowke. Predictions of arc temperature profiles using approximate emission coefficients for radiation losses. Journal of Quantitative Spectroscopy and Radiative Transfer, 14(2), 1974. doi: 10.1016/0022-4073(74)90004-1.

[15] R. Liebermann and J. Lowke. Radiation emission coefficients for sulfur hexafluoride arc plasma. JQSRT, 16(253), 1976. doi:10.1016/0022-4073(76) 90067-4

[16] V. Aubrecht and M. Bartlova. Net emission coefficients of radiation in air and $\mathrm{SF}_{6}$ thermal plasmas. Plasma Chemistry and Plasma Processing, 29(2):131, 2009. doi:10.1007/s11090-008-9163-x.

[17] A. Gleizes, B. Rahmani, J. Gonzalez, and B. Liani. Calculation of net emission coefficient in $\mathrm{N}_{2}, \mathrm{SF}_{6}$ and $\mathrm{SF}_{6}-\mathrm{N}_{2}$ arc plasmas. Journal of Physics D: Applied Physics, 24(8):1300, 1991. doi: $10.1088 / 0022-3727 / 24 / 8 / 011$.

[18] R. Siegel and J. Howell. Thermal radiation heat transfer. Third printing. Taylor and Francis, New York and London, 1992.

[19] M. Modest. Radiative Heat Transfer. Second printing. Academic Press California, London, Massachusetts, 2003.

[20] G. Raynal and A. Gleizes. Radiative transfer calculation in $\mathrm{SF}_{6}$ arc plasmas using partial characteristics. Plasma Sources and Technology, 4(1):152, 1995. doi:10.1088/0963-0252/4/1/017.

[21] V. Aubrecht and J. Lowke. Calculations of radiation transfer in $\mathrm{SF}_{6}$ plasmas using the method of partial characteristics. Journal of Physics D: Applied Physics, 27(10):2066, 1994. doi:10.1088/0022-3727/27/10/013.

[22] N. Bogatyreva, M. Bartlova, and V. Aubrecht. Mean absorption coefficients of air plasmas. Journal of Physics: Conference Series, 275(1), 2011. doi: 10.1088/1742-6596/275/1/012009.

[23] A. Gleizes, H. Randrianandraina, and Y. Cressault. Improvements of radiative transfer calculation for $\mathrm{SF}_{6}$ thermal plasmas. In XIXth Symposium on Physics of Switching arc, page 27, Brno, Czech Republic, 5-9 September 2011.

[24] T. Billoux, Y. Cressault, V. F. Boretskij, A. N. Veklich, and A. Gleizes. Net emission coefficient of $\mathrm{CO}_{2}-\mathrm{Cu}$ thermal plasmas: role of copper and molecules. J. Phys.: Conf. Ser., 406:012027, 2012. doi: 10.1088/1742-6596/406/1/012027.

[25] H. Z. Randrianandraina, Y. Cressault, and A. Gleizes. Improvements of radiative transfer calculation for $\mathrm{SF}_{6}$ thermal plasmas. J. Phys. D: Appl. Phys., 44(19):194012, 2011. doi:10.1088/0022-3727/44/19/194012.
[26] C. Jan, Y. Cressault, A. Gleizes, and K. Bousoltane. Calculation of radiative properties of $\mathrm{SF}_{6}-\mathrm{C}_{2} \mathrm{~F}_{4}$ thermal plasmas - application to radiative transfer in high-voltage circuit breakers modelling. J. Phys. D: Appl. Phys., 47(1):015204, 2013. doi:10.1088/0022-3727/47/1/015204.

[27] H. Nordborg and A. A. Lordanidis. Self-consistent radiation based modelling of electric arcs: I. Efficient radiation approximations. Journal of Physics D: Applied Physics, 41(13), 2008. doi : 10.1088/0022-3727/41/13/135205.

[28] L. Hermette, Y. Cressault, A. Gleizes, C. Jan, and $\mathrm{K}$. Bousoltane. Radiative properties of $\mathrm{SF}_{6}-\mathrm{C}_{2} \mathrm{~F}_{4}-\mathrm{Cu}$ mixtures in high voltage circuit breakers arc plasmas: net emission coefficient and mixing rules. In XXth International Conference on Gas Discharge and their Applications, page 229, Orléans, France, 6-11 July 2014.

[29] J. L. Zhang, A. B. Murphy, W. Hall, and M. T. C. Fang. Computational investigation of arc behavior in an auto-expansion circuit breaker contaminated by ablated nozzle vapor. IEEE Transactions on Plasma Science, 30(2):706-719, 2002. doi:10.1109/TPS .2002 .1024273.

[30] Q. Ma, M. Rong, A. B. Murphy, Y. Wu, and T. Xu. Simulation Study of the Influence of Wall Ablation on Arc Behavior in a Low-Voltage Circuit Breaker. IEEE Trans. Plasma Sci., 37(1):261-269, 2009. doi:10.1109/TPS.2008.2007733.

[31] J. Yan. Inclusion of nozzle dimensional change due to PTFE ablation in switching arc simulation. In XXIIth International Conference on Gas Discharge and their Applications, Novi-Sad, Serbia, 2-7 September 2018.

[32] P. Robin-Jouan. Ablation modelling in high voltage circuit-breakers: impact on the arc extinction. In XXth International Conference on Gas Discharge and their Applications, Orléans, France, 6-11 July 2014.

[33] S. Arabi, J. Trepanier, R. Camarero, and A. Vassilev. Modeling nozzle geometry changes due to the ablation in high-voltage circuit breakers. In XXth International Conference on Gas Discharge and their Applications, page 479, Orléans, France, 6-11 July 2014.

[34] A. Petchanka, F. Reichert, J. Gonzalez, and P. Freton. Improved modeling of ablation process in high voltage circuit breakers for switching ar simulation. In XXth International Conference on Gas Discharge and their Applications, page 155, Orléans, France, 6-11 July 2014.

[35] P. Kloc, V. Aubrecht, M. Bartlova, O. Coufal, and C. Rümpler. On the Selection of Integration Intervals for the Calculation of Mean Absorption Coefficients. Plasma Chem Plasma Process, 35(6):1097-1110, 2015. doi : 10.1007/s11090-015-9648-3.

[36] P. Kloc, V. Aubrecht, and M. Bartlova. Numerically optimized band boundaries of Planck mean absorption coefficients in air plasma. J. Phys. D: Appl. Phys., 50(30):305201, 2017. doi:10.1088/1361-6463/aa7627.

[37] P. Kloc, V. Aubrecht, and M. Bartlova. Objective function for numerical mean absorption bands optimization. Plasma Physics and Technology, 4(3):269, 2017. doi:10.14311/ppt.2017.3.269. 
[38] N. Kabbaj, Y. Cressault, P. Teulet, and F. Reichert. Numerical optimization of mean absorption coefficient in air using Planck modified mean function. In XVth Hig-Tech Plasma Processes Conference, Toulouse, France, 2-6 July 2018.

[39] J. P. Trelles. Advances and challenges in computational fluid dynamics of atmospheric pressure plasmas. Plasma Sources Sci. Technol., 27(9):093001, September 2018. doi:10.1088/1361-6595/aac9fa.

[40] A. Gleizes, F. Mbolidi, and A. A. M. Habib. Kinetic model of a decaying $\mathrm{SF}_{6}$ plasma over the temperature range $12000 \mathrm{~K}$ to $3000 \mathrm{~K}$. Plasma Sources Sci. Technol., 2(3):173-179, 1993. doi:10.1088/0963-0252/2/3/007.

[41] X. Baumann, Y. Cressault, P. Teulet, F. Reichert, and A. Petchanka. First approach in the calculation of the radiative properties of multi-temperature $\mathrm{SF}_{6}$ plasmas at 1 bar. In XXIIIrd International Symposium on Plasma Chemistry, Montréal, Canada, July 2017.

[42] X. Baumann. Etude de la composition chimique et des propriétés thermodynamiques et radiatives d'un plasma de $\mathrm{SF}_{6}-\mathrm{C}_{2} \mathrm{~F}_{4}$ en condition de déséquilibre thermique. PhD thesis, Université Paul Sabatier, France, 2019.

[43] V. Colombo, E. Ghedini, and P. Sanibondi. Thermodynamic and transport properties in non-equilibrium argon, oxygen and nitrogen thermal plasmas. Progress in Nuclear Energy, 50(8):921-933, 2008. doi:10.1016/j.pnucene.2008.06.002.

[44] V. Colombo, E. Ghedini, and P. Sanibondi. Two-temperature thermodynamic and transport properties of argon-hydrogen and nitrogen-hydrogen plasmas. J. Phys. D: Appl. Phys., 42(5):055213, 2009. doi:10.1088/0022-3727/42/5/055213.

[45] S. Ghorui, J. V. R. Heberlein, and E. Pfender. Thermodynamic and Transport Properties of Two-temperature Oxygen Plasmas. Plasma Chem Plasma Process, 27(3):267, 2007. doi:10.1007/s11090-007-9053-7.

[46] S. Ghorui, J. V. R. Heberlein, and E. Pfender. Thermodynamic and Transport Properties of Two-Temperature Nitrogen-Oxygen Plasma. Plasma Chem Plasma Process, 28(4):553-582, 2008. doi:10.1007/s11090-008-9141-3.

[47] X.-N. Zhang, H.-P. Li, A. B. Murphy, and W.-D. Xia. A numerical model of non-equilibrium thermal plasmas. I. Transport properties. Physics of Plasmas, 20(3):033508, 2013. doi:10.1063/1. 4794969.

[48] C. Bonnefois. Contribution à l'étude des méthodes de résolution de l'équation de Boltzmann dans un plasma à deux températures : exemple le mélange argon-hydrogène. $\mathrm{PhD}$ thesis, Limoges University, France, 1983.

[49] C. Bonnefoi, J. Aubreton, and J. M. Mexmain. New approach, taking into account elastic and inelastic processes for transport properties of a two temperature plasma. Zeitschrift für Naturforschung, 40:885, 1985. doi:10.1515/zna-1985-0904.

[50] J. Mexmain. Coefficients de transport dans un plasma à deux températures: application au mélange argon-oxygène. PhD thesis, 1983.
[51] F. McCourt. Nonequilibrium Phenomena in Polyatomic Gases: Dilute Gases. Vol. 1. Oxford University Press, USA, 1990.

[52] J. Aubreton, C. Bonnefoi, and J. M. Mexmain. Calcul de propriétés thermodynamiques et des coefficients de transport dans un plasma $\mathrm{Ar}-\mathrm{O}_{2}$ en non-équilibre thermodynamique et à la pression atmosphérique. Rev. Phys. Appl. (Paris), 21(6):365-376, 1986. doi:10.1051/rphysap:01986002106036500.

[53] J. Aubreton, M. F. Elchinger, and P. Fauchais. New Method to Calculate Thermodynamic and Transport Properties of a Multi-Temperature Plasma: Application to $\mathrm{N}_{2}$ Plasma. Plasma Chemistry and Plasma Processing, 18(1):1-27, 1998. doi:10.1023/A : 1021785125690.

[54] J. Aubreton, M. F. Elchinger, P. Fauchais, V. Rat, and P. André. Thermodynamic and transport properties of a ternary $\mathrm{Ar}-\mathrm{H}_{2}-\mathrm{He}$ mixture out of equilibrium up to $30000 \mathrm{~K}$ at atmospheric pressure. $J$. Phys. D: Appl. Phys., 37(16):2232-2246, 2004. doi : 10.1088/0022-3727/37/16/004.

[55] M. Capitelli, I. Armenise, D. Bruno, M. Cacciatore, R. Celiberto, G. Colonna, O. D. Pascale, P. Diomede, F. Esposito, C. Gorse, K. Hassouni, A. Laricchiuta, S. Longo, D. Pagano, D. Pietanza, and M. Rutigliano. Non-equilibrium plasma kinetics: a state-to-state approach. Plasma Sources Sci. Technol., 16(1):S30-S44, 2007. doi:10.1088/0963-0252/16/1/S03.

[56] M. Capitelli, I. Armenise, E. Bisceglie, D. Bruno, R. Celiberto, G. Colonna, G. D'Ammando, O. De Pascale, F. Esposito, C. Gorse, V. Laporta, and A. Laricchiuta. Thermodynamics, Transport and Kinetics of Equilibrium and Non-Equilibrium Plasmas: A State-to-State Approach. Plasma Chem Plasma Process, 32(3):427-450, 2012. doi: $10.1007 / \mathrm{s} 11090-011-9339-7$.

[57] X. Guo, A. B. Murphy, and X. Li. Thermodynamic properties and transport coefficients of two-temperature helium thermal plasmas. J. Phys. D: Appl. Phys., 50(12):125202, 2017. doi:10.1088/1361-6463/aa5d25.

[58] X. N. Zhang, H. P. Li, A. B. Murphy, and W. D. Xia. Comparison of the transport properties of twotemperature argon plasmas calculated using different methods. Plasma Sources Sci. Technol., 24(3):035011, 2015. doi:10.1088/0963-0252/24/3/035011.

[59] F. Yang, Z. Chen, Y. Wu, and al. Two-temperature transport coefficients of $\mathrm{SF}_{6}-\mathrm{N}_{2}$ plasma. Phys. Plasmas, 22(10), 2015. doi:10.1063/1.4932073.

[60] G. Vanhulle, Y. Cressault, P. Teulet, F. Reichert, and A. Petchanka. Transport properties of multi-temperature $\mathrm{SF}_{6}$ plasmas: influence of assumptions done in the plasma composition calculation. In XXIIIrd International Symposium on Plasma Chemistry, Montréal, Canada, 2017.

[61] L. Soucasse, P. Riviere, and A. Soufiani. Monte Carlo methods for radiative transfer in quasi-isothermal participating media. Journal of Quantitative Spectroscopy and Radiative Transfer, 128:34, 2014. doi:10.1016/j.jqsrt.2012.07.008.

[62] M. Scoggins. Development of numerical methods and study of coupled flow, radiation, and ablation phenomena for atmospheric entry. $\mathrm{PhD}$ thesis, Univsersity Paris-Saclay, 2017. 